\title{
Mid-basal left ventricular longitudinal dysfunction as a prognostic marker in mutated transthyretin-related cardiac amyloidosis
}

\author{
Roberto Licordari', Fabio Minutoli², Francesco Cappelli ${ }^{3}$, Antonio Micari' ${ }^{2}$, Luigi Colarusso', Federico \\ Antonio Francesco Di Paola ${ }^{1}$, Mariapaola Campisi ${ }^{1}$, Antonino Recupero ${ }^{1}$, Anna Mazzeo ${ }^{4}$, Gianluca Di Bella ${ }^{1}$ \\ ${ }^{1}$ Rare Cardiac Disease Centre, Department of Clinical and Experimental Medicine, University of Messina, Messina 98125, Italy. \\ ${ }^{2}$ Department of Biomedical Sciences and Morphologic and Functional Images, University of Messina, Messina 98125, Italy. \\ ${ }^{3}$ Tuscan regional amyloid center, Careggi University Hospital, Firenze 50134, Italy. \\ ${ }^{4}$ Neurology Unit, Department of Clinical and Experimental Medicine, University of Messina, Messina 98125, Italy.
}

Correspondence to: Dr. Roberto Licordari, Rare Cardiac Disease Centre, Department of Clinical and Experimental Medicine, Via Consolare Valeria 1, University of Messina, Messina 98125, Italy. E-mail: robertolicordari@gmail.com

\begin{abstract}
How to cite this article: Licordari R, Minutoli F, Cappelli F, Micari A, Colarusso L, Di Paola FAF, Campisi M, Recupero A, Mazzeo A, Di Bella G. Mid-basal left ventricular longitudinal dysfunction as a prognostic marker in mutated transthyretin-related cardiac amyloidosis. Vessel Plus 2022;6:12. https://dx.doi.org/10.20517/2574-1209.2021.86
\end{abstract}

Received: 11 Jun 2021 First Decision: 17 Aug 2021 Revised: 26 Aug 2021 Accepted: 12 Oct 2021 Published: 17 Feb 2022

Academic Editors: Alexander D. Verin, Ugolino Livi, Gianfranco Sinagra Copy Editor: Yue-Yue Zhang Production Editor: YueYue Zhang

\begin{abstract}
Aim: Mutated transthyretin (TTRv) cardiac amyloidosis (CA) represents an uncommon form of CA. Our study aimed to assess the best echocardiographic prognostic parameter in the early stage of TTRv amyloidosis with cardiomyopathy.

Methods: In total, 99 patients with TTRv in New York Heart Association class I or II and with no clinical history of previous cardiac disease were studied. Assessment with ${ }^{99 m} \mathrm{Tc}$-DPD whole-body scan showed CA in 46 patients. At the first medical contact, an echocardiographic examination was performed. In addition to conventional left ventricular (LV), echocardiographic measurements [ejection fraction (EF), dimensions and diastolic function, global longitudinal strain (GLS), longitudinal strain of the 4 apical segments, longitudinal strain of the 12 mid-basal segments (MBLS)] and their ratio [relative regional strain ratio (RRSR)] were obtained. Patient outcome was evaluated during a follow-up with an average duration of six years. Cardiac death and appropriate implantable cardiac defibrillator (ICD) shock were considered major events.
\end{abstract}


Results: A higher value of LV thickness, E/E', and RRSR and lower LV volumes, EF, GLS, MBLS were observed in patients with CA than the ones with only TTRv. During follow-up (median of 7.7 years), 25 major events (23 cardiac death and 2 appropriate ICD shocks) occurred. The logistic univariate analyses showed that LV EF, LV septal thickness, E/E', GLS, and MBLS were all predictors of cardiovascular death. In multivariate analysis, MBLS was the only independent predictor of major events. A cut off of - 14 of MBLS was selected as the best value to discriminate a worse prognosis on Kaplan-Meyer analysis.

Conclusion: Longitudinal dysfunction is observed in the early stages of ATTRv amyloidosis with cardiomyopathy. Medio-basal LV longitudinal dysfunction is a strong independent echocardiographic predictor of cardiac death.

Keywords: Cardiac amyloidosis, myocardial strain imaging, prognosis

\section{INTRODUCTION}

Familial transthyretin amyloidosis due to a mutation of the gene coding for transthyretin (TTRv) is one of the three most frequent subtypes, together with light chain (AL) and wild-type TTR amyloidosis (wtTTR) ${ }^{[1-3]}$. Among these, mutated TTR represents the most uncommon form of cardiac amyloidosis (CA).

In TTRv patients, penetrance and disease phenotype is variable according to the different well-known TTR gene mutation $s^{[4]}$. In the clinical management of carriers, the identification of early cardiac involvement, ideally in a presymptomatic stage, is of paramount importance to achieve correct management and early treatment.

Non-invasive diagnosis of CA is performed using an integrated evaluation of biomarkers (troponins and natriuretic peptides), echocardiography, cardiac magnetic resonance, and ${ }^{99 m} \mathrm{Tc}-3,3$-diphosphono-1,2 propanodicarboxylic acid (DPD) scintigraphy. DPD scintigraphy, identifying with high accuracy TTRrelated amyloidosis, is considered the non-invasive gold standard diagnostic tool for ATTR amyloidosis with cardiomyopathy ${ }^{[5-7]}$.

In clinical practice, echocardiography is the imaging tool most used during both initial evaluation and follow-up of CA patients. A relative "apical sparing" pattern of longitudinal deformation is an easily recognizable, accurate, and reproducible method of differentiating CA from other causes of left ventricular (LV) hypertrophy ${ }^{[8]}$.

Echocardiographic parameters such as increased LV wall thickness, global longitudinal strain (GLS), reduced ejection fraction (EF), and a high relative regional strain ratio are independent predictors of cardiac mortality in AL amyloidosis ${ }^{[9]}$.

On the contrary, no large studies have evaluated the prognostic role of an integrated echocardiographic approach in patients with ATTR amyloidosis with cardiomyopathy especially in the early stage of the disease.

Our study aimed to assess the best conventional and strain-derived echocardiographic prognostic parameters in the early clinical stage of ATTRv amyloidosis with cardiomyopathy. 


\section{METHODS}

In total, 119 patients (60 men and 59 women; mean age $48 \pm 11$ years), belonging to seven unrelated families with TTR gene mutation (Glu89Gln, Phe64Leu, and Thr49Ala) followed at the Department of Cardiology of University Hospital of Messina, were prospectively enrolled from June 2010 to February 2018. None of the included patients had evidence of monoclonal protein in the serum or urine, a monoclonal population of plasma cells in the bone marrow, or other diseases that could be responsible for secondary amyloidosis.

All patients underwent the following examinations: two-dimensional standard echocardiography, strain echocardiography, and ${ }^{99 m}$ Tc-DPD scan. Patients with New York Heart Association (NYHA) III and IV and/or previous cardiac disease were excluded.

The study was approved by our institutional review board. Informed consent was obtained from all patients.

\section{Standard echocardiography data acquisition and analysis}

Standard echocardiographic examinations were performed in all patients using a commercial ultrasound machine (My Lab ALFA, Esaote, Florence, Italy) equipped with a $2.5 \mathrm{MHz}$ phased-array transducer. Parasternal short-axis views at the basal, mid, and apical levels and three standard apical views (4-chamber, 2-chamber, and LV outflow long-axis) were acquired. The same cardiologist performed all examinations. The following measurements were obtained according to recommendations of the American Society for Echocardiography: diastolic thickness of the LV basal anterior septum (LVST), basal posterior wall thickness, LV volumes (end-diastolic volume and end-systolic volume), EF, and LV diastolic function quantified by the ratio between the E-wave velocity of the pulsed-wave Doppler mitral flow image and the early diastolic velocity of the septum at the mitral annulus level (E’ wave) on tissue Doppler imaging ${ }^{[10,11]}$.

\section{Strain acquisition and analysis}

A dedicated software package (XStrain ${ }^{\text {tw }}$, Esaote, Florence, Italy) was used for an offline quantification of right and left strain. A 16-segment model was used to divide the LV. Left ventricular longitudinal strain was acquired on 4- and 2-chamber apical views. GLS was obtained from the average longitudinal strain of the 16 segments on apical views; apical longitudinal strain (ALS) as the average of 4 apical segments and mediobasal longitudinal strain (MBLS) as the average of the 12 medial and basal segments. Relative regional strain ratio (RRSR) was calculated by dividing the average apical LS by the sum of the basal and mid-LS values ${ }^{[9]}$. Strain analysis was performed by a single trained cardiologist, blinded to patient's clinical data. Later, another trained cardiologist, blinded to the previous measurements, performed the same analysis on 16 patients to test the inter-observer reliability.

\section{${ }^{99 m}$ TC-DPD data acquisition and analysis}

Whole-body scans (anterior and posterior projections) were obtained $5 \mathrm{~min}$ and $3 \mathrm{~h}$ after the intravenous injection of $740 \mathrm{MBq}$ of ${ }^{99 \mathrm{~m}} \mathrm{Tc}-\mathrm{DPD}$ using a dual-headed gamma camera (MillenniumVG, GE Healthcare, Milwaukee, Wisconsin, USA) equipped with low-energy, high-resolution collimators.

The whole-body scans were visually evaluated by consensus of two experienced nuclear medicine physicians who searched for cardiac radiotracer accumulation; readers were blinded to echocardiographic data.

A semi-quantitative score was obtained based on the results of planar images as previously described by Perugini ${ }^{[12]}$. Briefly, a score of 0 signified absence of cardiac uptake, 1 indicated mild uptake less than bone, 2 indicated moderate uptake equal to the bone, and 3 indicated strong uptake with mild or absent bone uptake. 
Readers evaluated the eventual presence of cardiac radiotracer accumulation as positive when a semiquantitative score of 2 or 3 was found.

\section{Follow-up}

Patient outcome was evaluated from the day of the first visit to the day of the last medical contact (periodic ambulatory work up in our institute or telephone contact). The major events considered were cardiac death and appropriate implantable cardiac defibrillator (ICD) shock. The cause of death was derived from medical records or death certificates. The definition of cardiac death required the documentation of significant arrhythmia or cardiac arrest or death attributable to congestive heart failure. In the case of out-of-hospital death not followed by autopsy, sudden unexpected death was classified as cardiac death. Implantable cardiac defibrillator shocks were designated appropriate if triggered by lethal arrhythmias: ventricular tachycardia above the programmed cut-off of the ICD (12 intervals at 180 b.p.m.) or ventricular fibrillation. A complete interrogation of the ICD was performed by the referring physician to confirm the appropriateness of the $\operatorname{shock}^{[13]}$.

All cardiovascular events were assessed by two independent cardiologists, who were blinded to clinical and echocardiographic patients' data.

\section{Statistical analysis}

Continuous variables were expressed as mean $+\mathrm{SD}$ or median (25th; 75th percentiles). Categorical variables were expressed as a percentage. The comparison between continuous variables in patients with and without cardiac events was performed by Student's independent $t$-test or the Wilcoxon test as appropriate. A Cox regression analysis was performed for the identification of predictors of cardiovascular mortality-free survival. Receiver operating characteristic (ROC) curves were performed to find the best cut-off by sensitivity and specificity for variables. The Kaplan-Meier curves were processed, with the log-rank test for the comparison of survival curves. $P$-value $\leq 0.05$ was considered significant. Statistical analysis was performed using dedicated software (IBM SPSS Statistics, v 26.0, Chicago, Illinois).

\section{RESULTS}

In total, 119 patients were identified; 20 patients were excluded according to the presence of previous heart diseases (previous myocardial infarction in 1 patient and moderate-severe aortic stenoses in 5 patients) and NYHA $\geq$ III (14 patients). Therefore, our population consists of 99 patients with TTRv ( 45 Glu89Gln, 40 Phe64Leu, and 14 Thr49Ala) in NYHA functional class I or II and had no clinical history of previous cardiac disease.

In all 99 patients, ${ }^{99 m}$ Tc-DPD whole-body scan was performed and radiotracer accumulation (Perugini score) showing CA was found in 46 subjects (19 female) [Figure 1].

\section{Echocardiographic findings: LV dimensions and function}

The CA group showed higher values of interventricular septal (IVS) thickness, LV posterior wall thickness, and $\mathrm{E} / \mathrm{E}$ ' than the control group. LVEF was significantly lower, but still within the normal range, in the CA group than the no-CA group [Table 1].

GLS, MBLS, and RRSR of the LV were higher in the CA group than the control group, while ALS was similar in the CA group and the control [Table 1]. 
Table 1. Demographic, clinical, and echocardiographic variables in CA and control group

\begin{tabular}{llll}
\hline & CA group $(\boldsymbol{n}=\mathbf{4 6})$ & Mutated TTR patients without CA controls $(\boldsymbol{n}=\mathbf{5 3})$ & $\boldsymbol{P}$-value \\
\hline Age (years) & $50 \pm 10.2$ & $47 \pm 9$ & 0.22 \\
Heart rate (bpm) & $77 \pm 11$ & $75.6 \pm 8.7$ & 0.63 \\
BSA & $1.73 \pm 0.3$ & $1.76 \pm 0.4$ & 0.78 \\
Mean systolic blood pressure (mmHg) & $120 \pm 18$ & $122 \pm 19$ & 0.01 \\
BNP (pg/mL) & $1172.6 \pm 3233$ & $63 \pm 29.5$ & 0.04 \\
LV EDV (mL) & $83 \pm 20.5$ & $94.7 \pm 29.5$ & 0.83 \\
LV ESV (mL) & $34.3 \pm 12.6$ & $35 \pm 12.8$ & 0.33 \\
LV stroke volume (mL) & $49.3 \pm 13.4$ & $53.2 \pm 19.7$ & 0.23 \\
LV stroke volume index (mL/m $\left.{ }^{2}\right)$ & $28.5 \pm 7$ & $30.2 \pm 7$ & $<0.001$ \\
LV EF (\%) & $56.7 \pm 12$ & $68 \pm 5$ & $<0.001$ \\
LV ST (mm) & $13.9 \pm 3.2$ & $8.7 \pm 0.5$ & $<0.001$ \\
LV posterior wall (mm) & $12 \pm 3$ & $8.3 \pm 1.1$ & $<0.001$ \\
E/E' & $9.3 \pm 5.3$ & $4.7 \pm 0.9$ & 0.04 \\
GLS (\%) & $-15.5 \pm 5$ & $-18 \pm 3$ & 0.94 \\
ALS (\%) & $-16.4 \pm 5.7$ & $-16.5 \pm 2.5$ & $<0.001$ \\
MLS (\%) & $-14.2 \pm 5.5$ & $-18.3 \pm 0.8$ & 0.01 \\
RRSR & $1.25 \pm 0.55$ & $0.91 \pm 0.15$ & $<0.001$ \\
SPAP (mmHg) & $30.6 \pm 8.3$ & $27.7 \pm 2.5$ & 0.04 \\
TAPSE (mm) & $17 \pm 4$ & $19.5 \pm 1.5$ & \\
\hline
\end{tabular}

BSA: Body surface area; BNP: nrain natriuretic peptide; LV EDV: left ventricle end diastolic volum; LV ESV: left ventricle end systolic volum; LV EF: left ventricle ejection fraction; LV EDD: left ventricle end diastolic diameter; LV ESD: left ventricle end diastolic diameter; LV ST: left ventricle septal thickness; GLS: global longitudinal strai; ALS: apical longitudinal strain; MBLS: mid-basal longitudinal strain; RRSR: relative regional strain ratio; sPAP: systolic pulmonary pressure.

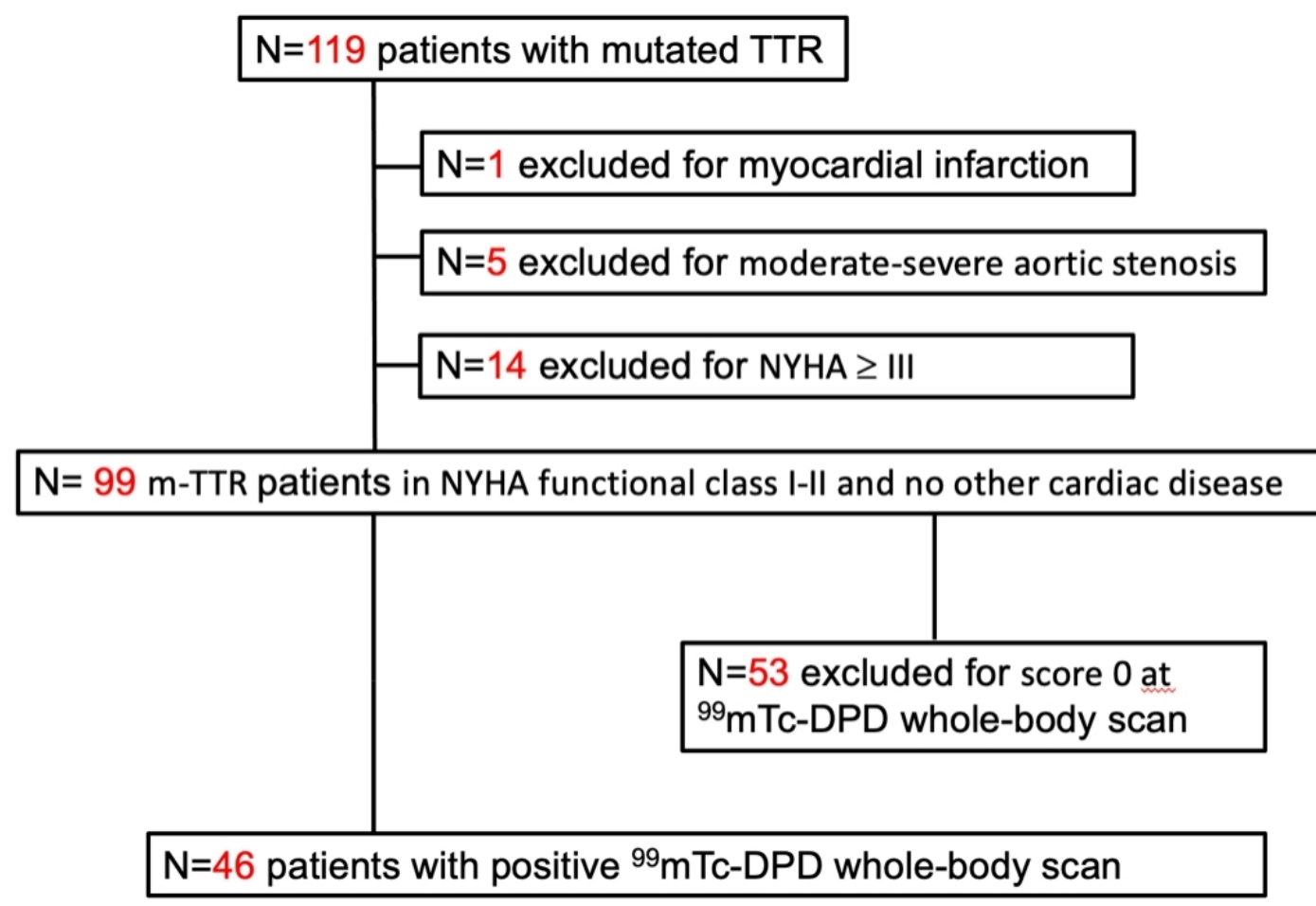

Figure 1. Study population: flow chart visualizing the derivation of the study population. 
Data on the inter-observer consistency for myocardial strain analysis are reported in Supplementary Table 1.

\section{Cardiac endpoint}

The average duration of follow-up was 6.5 years (7.7 median, range 2-9.8 years). Major events occurred in 25 patients (54\%), cardiac events in 23 patients (50\%) and ICD shocks in 2 . As reported in Table 2 , both systolic and diastolic functions were significantly worse in patients with major cardiac events. Left ventricular posterior wall thickness and IVS were higher in patients with major cardiac events compared to those without. Patients with major cardiac events also showed lower GLS, ALS, and MBLS compared to survivors.

The logistic analysis [Table 3] showed that LV EF, LVST, E/E', GLS, and MBLS were all predictors of cardiovascular death in univariate analyses.

The multivariate analysis showed that, among eco-derived variables, MBLS was an independent predictor of mortality [Table 3].

According to ROC analysis, a cut-off of -14 of MBLS was the best result in terms of combined sensitivity and specificity in identifying major cardiac events on follow-up (sensitivity 100\%, specificity $70 \%$, with an AUC 0.81).

The patients were divided into two groups based on the previous MBLS value found by ROC curve. KaplanMeyer analysis across the TTRm CA population showed a significantly reduced survival in patients with a MBLS lower than $-14(P<0.001)$ [Figure 2].

\section{DISCUSSION}

The main results of the present study are: (1) longitudinal dysfunction is observed in TTRv patients with CA in the early stages; (2) mediobasal LV longitudinal dysfunction is the best echocardiographic predictor of cardiac death in ATTRv amyloidosis with cardiomyopathy; and (3) a relative apical sparing of strain is not a predictor of cardiac death in TTRv CA in patients with non-severe heart failure (NYHA I-II) and no other cardiac disease.

In the present study, we showed that strain imaging is not only useful for diagnosis, but it is a powerful prognostic indicator of long-term survival even if applied in the subset of early stages ATTRv amyloidosis with cardiomyopathy.

Previous studies demonstrated the value of tissue Doppler and strain imaging for diagnosis and staging of cardiac dysfunction in patients with $\mathrm{CA}^{[14-16]}$ as well in other systemic diseases with cardiac involvement ${ }^{[17]}$. Global longitudinal strain and apical sparing have been shown to be an accurate diagnostic marker of CA.

Particularly, apical sparing permits discriminating CA in the context of hypertrophic phenotypes cardiomyopathies $^{[18]}$.

A relative apical sparing of strain has been reported as the only independent predictor of major adverse cardiac events in AL CA ${ }^{[8,9,19]}$. Senapati et al. ${ }^{[9]}$ studied 97 patients with a definite diagnosis of CA (59 AL, 23 wtTTR, and 15 TTRv) and found that, among predictors (AL subtype, EF, and NYHA class $\geq$ III), the ratio between apical and mid-basal segments remains independently predictive of all-cause mortality or heart 
Table 2. Differences in variables between patients who presented major cardiac events during follow-up and patients who are still alive

\begin{tabular}{llll}
\hline & Presence of major cardiac events $(\boldsymbol{n}=\mathbf{2 5}$ pts $)$ & Absence of major cardiac events $(\boldsymbol{n}=\mathbf{2 1}$ pts $)$ & P-value \\
\hline LV EDV (mL) & $85 \pm 21$ & $82 \pm 20$ & 0.57 \\
LV ESV (mL) & $37.5 \pm 14.5$ & $32 \pm 11$ & 0.13 \\
LV EF (\%) & $48 \pm 7.5$ & $60 \pm 12$ & $\mathbf{0 . 0 1}$ \\
LV EDD (mm) & $50 \pm 9.5$ & $49 \pm 7.5$ & 0.57 \\
LV ESD (mm) & $31 \pm 8.3$ & $30 \pm 7$ & 0.63 \\
LV ST (mm) & $16.2 \pm 2.8$ & $12.3 \pm 2.5$ & $<\mathbf{0 . 0 0 1}$ \\
LV posterior wall (mm) & $14 \pm 2.5$ & $10.5 \pm 2.5$ & $<\mathbf{0 . 0 1}$ \\
E/E' & $13 \pm 5$ & $7 \pm 4$ & $<\mathbf{0 . 0 0 1}$ \\
GLS (-\%) & $11.5 \pm 3$ & $19 \pm 4$ & $<\mathbf{0 . 0 0 1}$ \\
ALS (-\%) & $13.5 \pm 6.7$ & $18 \pm 4$ & $\mathbf{0 . 0 1}$ \\
MBLS (-\%) & $10.5 \pm 2.5$ & $16.5 \pm 5.3$ & $<\mathbf{0 . 0 1}$ \\
RRSR & $1.32 \pm 0.7$ & $1.20 \pm 0.4$ & 0.50 \\
\hline
\end{tabular}

LV EDV: Left ventricle end diastolic volum; LV ESV: left ventricle end systolic volum; LV EF: left ventricle ejection fraction; LV EDD: left ventricle end diastolic diameter; LV ESD: left ventricle end diastolic diameter; LV ST: left ventricle septal thickness; GLS: global longitudinal strain; ALS: apical longitudinal strain; MBLS: mid-basal longitudinal strain; RRSR: relative regional strain ratio.

Table 3. Univariate and multivariate analysis in identifying predictors of mortality in cardiac amyloidosis

\begin{tabular}{|c|c|c|c|c|c|c|c|c|}
\hline & \multicolumn{2}{|c|}{ Univariate analysis } & \multicolumn{2}{|c|}{ Multivariate analysis } & \multicolumn{2}{|c|}{ Multivariate analysis } & \multicolumn{2}{|c|}{ Multivariate analysis } \\
\hline & $\mathrm{HR}(95 \% \mathrm{Cl})$ & $P$-value & $\mathrm{HR}(95 \% \mathrm{Cl})$ & $P$-value & $\mathrm{HR}(95 \% \mathrm{Cl})$ & $P$-value & $\mathrm{HR}(95 \% \mathrm{Cl})$ & $P$-value \\
\hline Age & $1.02(0.97-1.08)$ & 0.29 & & & & & & \\
\hline LV EDV $(m L)$ & $0.99(0.97-1.01)$ & 0.35 & & & & & & \\
\hline LV ESV (mL) & $1.01(0.98-1.04)$ & 0.39 & & & & & & \\
\hline LV septal thickness (mm) & $1.17(1.03-1.32)$ & 0.01 & $0.12(0.01-1.89)$ & 0.13 & $1.37(1.00-1.87)$ & 0.06 & $1.39(0.95-2.05)$ & 0.09 \\
\hline LV EF (\%) & $0.83(0.70-0.99)$ & 0.04 & $0.76(0.54-1.06)$ & 0.11 & & & & \\
\hline$E / E^{\prime}$ & $1.15(1.06-1.25)$ & 0.001 & $6.30(0.71-55.35)$ & 0.10 & $0.83(0.67-1.04)$ & 0.11 & $0.85(0.64-1.13)$ & 0.27 \\
\hline GLS (\%) & $0.86(0.76-0.97)$ & 0.01 & & & $0.88(0.73-1.06)$ & 0.18 & & \\
\hline ALS (\%) & $0.96(0.89-1.02)$ & 0.15 & & & & & & \\
\hline MBLS (\%) & $0.85(0.71-0.96)$ & 0.01 & & & & & $0.84(0.71-0.98)$ & 0.04 \\
\hline RRSR & $1.37(0.64-2.99)$ & 0.42 & & & & & & \\
\hline
\end{tabular}

LV EDV: Left ventricle end diastolic volume; LV ESV: left ventricle end systolic volume; LV EF: left ventricle ejection fraction; GLS: global longitudinal strain; ALS: apical longitudinal strain; MBLS: mid-basal longitudinal strain; RRSR: relative regional strain ratio.

transplantation. Interestingly, Senapati et al. ${ }^{[9]}$ showed a predictive value of mortality according to the ratio between apical and mid-basal segments particularly in patients with EF lower than 45\%. Differently from Senapati's study ${ }^{[9]}$, we enrolled a homogenous cohort formed by early-stage TTRm CA who showed preserved EF.

The clinical evolution and follow-up of ATTRv amyloidosis with cardiomyopathy are very different compared to AL CA.

Light-chain amyloidosis is associated with rapidly progressive disease due to cardiotoxic effects with a diffuse impairment of myocardial deformation. On the other hand, TTR amyloidosis, although it tended to have higher mass and wall thickness, had a mild depression of LV systolic function. 


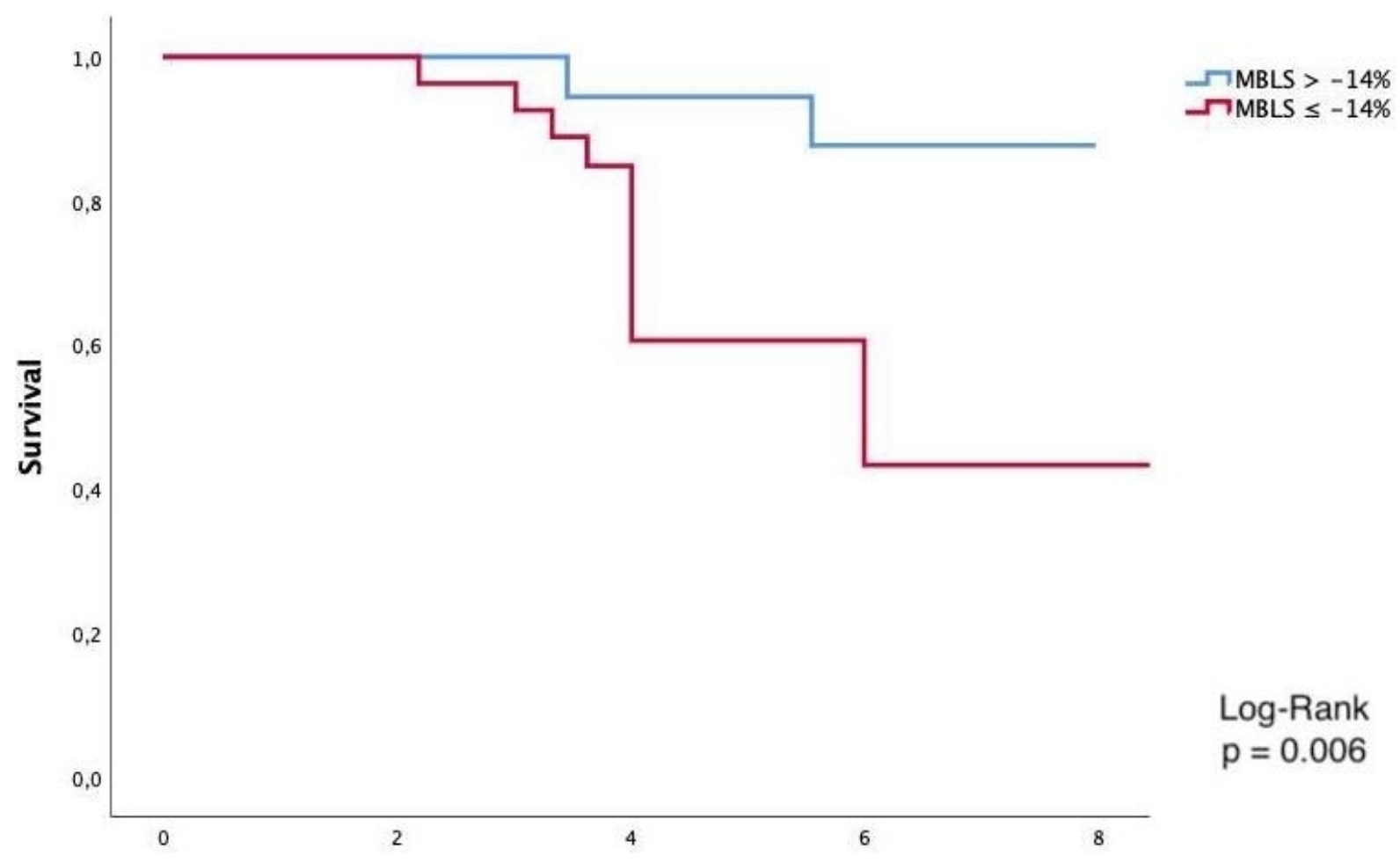

Figure 2. Kaplan-Meyer analysis of mortality in patients with a MBLS > -14 (blue line) and patients with a MBLS $\leq-14$ (red line).

LV longitudinal deformation becomes more impaired beginning in the basal and mid segments and progresses to involve distal LV segments.

GLS was not proven as an independent predictor of mortality in previous studies ${ }^{[20]}$. This is due to the compensative value of preserved apical deformation on the global value of LV deformation.

In the present study, we demonstrated that MBLS, avoiding apical segments, can be a helpful independent predictor of cardiovascular mortality over time, especially in patients in the early stages of TTRv amyloidosis with cardiomyopathy.

Our results extend and confirm the research of Siepen et al. ${ }^{[20]}$, in which impairment of mid-ventricular deformation was a strong adverse predictor of survival in TTRwt amyloidosis with cardiomyopathy.

\section{Limitations}

A major limitation of our study was the small number of patients. However, the prevalence of the disease is very low and collecting a larger population is challenging. Patients may have received interim therapies that may have affected prognosis.

Another major weakness was the lack of cardiac biopsy specimens available for histopathology. However, this procedure could not be indicated in our asymptomatic patients. Nevertheless, the existence of previous studies on the histopathologic changes of CA allows us to hypothesize reliably on the pathologic abnormalities that may correlate with and explain the imaging findings. 


\section{Conclusion}

Systolic longitudinal function is impaired early in ATTRv amyloidosis with cardiomyopathy, and this alteration is related to abnormalities of LV morphology and function. Impairment of systolic function in mediobasal LV segments shows a great prognostic role in predicting cardiovascular mortality over followup. Further studies in a larger sample of patients with amyloid deposition are needed to confirm our results.

\section{DECLARATIONS}

\section{Authors' contributions}

Writing original draft, formal analysis, data curation, visualization: Licordari R, Di Bella G

Conceptualization, funding acquisition, writing, review and editing, supervision: Colarusso L, Di Paola FAF, Campisi $\mathrm{M}$

Writing, review and editing, investigation, and validation: Minutoli F, Cappelli F, Micari A, Recupero A, Mazzeo A

\section{Availability of data and materials}

Not applicable

\section{Financial support and sponsorship}

None.

\section{Conflicts of interest}

All authors declared that there are no conflicts of interest.

\section{Ethical approval and consent to participate}

Not applicable.

\section{Consent for publication}

Not applicable.

\section{Copyright}

(c) The Author(s) 2022.

\section{REFERENCES}

1. Merlini G, Seldin DC, Gertz MA. Amyloidosis: pathogenesis and new therapeutic options. J Clin Oncol 2011;29:1924-33. DOI PubMed PMC

2. Sipe JD, Benson MD, Buxbaum JN, et al. Amyloid fibril protein nomenclature: 2010 recommendations from the nomenclature committee of the International Society of Amyloidosis. Amyloid 2010;17:101-4. DOI PubMed

3. Mazzeo A, Russo M, Di Bella G, et al. Transthyretin-related familial amyloid polyneuropathy (TTR-FAP): a single-center experience in sicily, an italian endemic area. JNeuromuscul Dis 2015;2:S39-48. DOI PubMed PMC

4. Koike H, Katsuno M. Transthyretin amyloidosis: update on the clinical spectrum, pathogenesis, and disease-modifying therapies. Neurol Ther 2020;9:317-33. DOI PubMed PMC

5. Minutoli F, Di Bella G, Sindoni A, Vita G, Baldari S. Effectiveness of skeletal scintigraphy in transthyretin-related amyloidosis. Int $J$ Cardiol 2013;168:4988-9. DOI PubMed

6. Di Bella G, Minutoli F, Mazzeo A, et al. MRI of cardiac involvement in transthyretin familial amyloid polyneuropathy. AJR Am $J$ Roentgenol 2010;195:W394-9. DOI PubMed

7. Koike H, Okumura T, Murohara T, Katsuno M. Multidisciplinary approaches for transthyretin amyloidosis. Cardiol Ther 2021. DOI PubMed PMC

8. Phelan D, Collier P, Thavendiranathan P, et al. Relative apical sparing of longitudinal strain using two-dimensional speckle-tracking echocardiography is both sensitive and specific for the diagnosis of cardiac amyloidosis. Heart 2012;98:1442-8. DOI PubMed

9. Senapati A, Sperry BW, Grodin JL, et al. Prognostic implication of relative regional strain ratio in cardiac amyloidosis. Heart 2016;102:748-54. DOI PubMed

10. Nagueh SF, Middleton KJ, Kopelen HA, Zoghbi WA, Quiñones MA. Doppler tissue imaging: a noninvasive technique for evaluation of left ventricular relaxation and estimation of filling pressures. J Am Coll Cardiol 1997;30:1527-33. DOI PubMed

11. Rudski LG, Lai WW, Afilalo J, et al. Guidelines for the echocardiographic assessment of the right heart in adults: a report from the 
American Society of Echocardiography endorsed by the European Association of Echocardiography, a registered branch of the European Society of Cardiology, and the Canadian Society of Echocardiography. J Am Soc Echocardiogr 2010;23:685-713; quiz 7868. DOI PubMed

12. Perugini E, Guidalotti PL, Salvi F, et al. Noninvasive etiologic diagnosis of cardiac amyloidosis using 99mTc-3,3-diphosphono-1,2propanodicarboxylic acid scintigraphy. J Am Coll Cardiol 2005;46:1076-84. DOI PubMed

13. Di Bella G, Siciliano V, Aquaro GD, et al. Scar extent, left ventricular end-diastolic volume, and wall motion abnormalities identify high-risk patients with previous myocardial infarction: a multiparametric approach for prognostic stratification. Eur Heart $J$ 2013;34:104-11. DOI PubMed

14. Bellavia D, Abraham TP, Pellikka PA, et al. Detection of left ventricular systolic dysfunction in cardiac amyloidosis with strain rate echocardiography. J Am Soc Echocardiogr 2007;20:1194-202. DOI PubMed

15. Bellavia D, Pellikka PA, Abraham TP, et al. Evidence of impaired left ventricular systolic function by Doppler myocardial imaging in patients with systemic amyloidosis and no evidence of cardiac involvement by standard two-dimensional and Doppler echocardiography. Am J Cardiol 2008;101:1039-45. DOI PubMed

16. Koyama J, Ray-Sequin PA, Falk RH. Longitudinal myocardial function assessed by tissue velocity, strain, and strain rate tissue Doppler echocardiography in patients with AL (primary) cardiac amyloidosis. Circulation 2003;107:2446-52. DOI PubMed

17. Karavidas A, Xylomenos G, Matzaraki V, et al. Myocardial deformation imaging unmasks subtle left ventricular systolic dysfunction in asymptomatic and treatment-naïve HIV patients. Clin Res Cardiol 2015;104:975-81. DOI PubMed

18. Di Bella G, Minutoli F, Pingitore A, et al. Endocardial and epicardial deformations in cardiac amyloidosis and hypertrophic cardiomyopathy. Circ J 2011;75:1200-8. DOI PubMed

19. Ternacle J, Bodez D, Guellich A, et al. Causes and consequences of longitudinal LV dysfunction assessed by 2D strain echocardiography in cardiac amyloidosis. JACC Cardiovasc Imaging 2016;9:126-38. DOI PubMed

20. Siepen FAD, Bauer R, Voss A, et al. Predictors of survival stratification in patients with wild-type cardiac amyloidosis. Clin Res Cardiol 2018;107:158-69. DOI PubMed 